\title{
ANALYSES OF SEPARATE AND CONCATENATED COX1 AND 18S rRNA GENE SEQUENCES INDICATE THAT THE BAT PIROPLASM BABESIA VESPERUGINIS IS PHYLOGENETICALLY CLOSE TO CYTAUXZOON FELIS AND THE 'PROTOTHEILERID' BABESIA CONRADAE
}

\author{
Sándor HORNOK ${ }^{1 *}$, Alexandra CORDUNEANU ${ }^{2}$, Jenö KONTSCHÁN ${ }^{3}$, Katinka BEKÖ ${ }^{4}$, \\ Krisztina SzÖKE ${ }^{1}$, Tamás GÖRFÖL ${ }^{5}$, Miklós GYURANECZ ${ }^{4}$ and Attila D. SÁNDOR ${ }^{2}$ \\ ${ }^{1}$ Department of Parasitology and Zoology, University of Veterinary Medicine, István u. 2, \\ H-1078 Budapest, Hungary; ${ }^{2}$ Department of Parasitology and Parasitic Diseases, \\ University of Agricultural Sciences and Veterinary Medicine, Cluj-Napoca, Romania; \\ ${ }^{3}$ Plant Protection Institute, Centre for Agricultural Research, Hungarian Academy of \\ Sciences, Budapest, Hungary; ${ }^{4}$ Institute for Veterinary Medical Research, Centre for \\ Agricultural Research, Hungarian Academy of Sciences, Budapest, Hungary; \\ ${ }^{5}$ Department of Zoology, Hungarian Natural History Museum, Budapest, Hungary
}

(Received 7 September 2017; accepted 12 February 2018)

\begin{abstract}
Babesia vesperuginis is the only piroplasm known to infect bats. Unlike most members of the genus Babesia, it is probably transmitted by a soft tick species (i.e. Argas vespertilionis). Recently, two studies have been conducted to clarify the phylogenetic status of this species, and both agreed on placing it into a basal position among Babesia sensu stricto (s.s.). However, several important groups of piroplasms were not included in the already reported phylogenetic trees of $B$. vesperuginis isolates. Therefore, the aim of the present study was to amplify an approx. 950-bp fragment of the cytochrome $c$ oxidase subunit 1 (coxl) gene of $B$. vesperuginis from $A$. vespertilionis specimens, and to compare its sequences with those from other piroplasmid groups in a broader phylogenetic context. Sequence comparisons focusing on either $18 \mathrm{~S}$ rRNA or coxl genes, as well as phylogenetic analyses involving separate and concatenated 18S rRNA and coxl sequences indicate that $B$. vesperuginis is more closely related to the phylogenetic group of Theileriidae than to Babesia s.s. In particular, B. vesperuginis clustered closest to Cytauxzoon felis and the 'prototheilerid' B. conradae. The results of this study highlight that $B$. vesperuginis is a unique and taxonomically important species, which should be included in future studies aimed at resolving the comprehensive phylogeny of Piroplasmida.
\end{abstract}

Key words: Apicomplexa, Piroplasmida, Western Babesia group, Chiroptera

*Corresponding author; E-mail: Hornok.Sandor@univet.hu; Phone: 0036 (1) 478-4187

Open Access. This is an open-access article distributed under the terms of the Creative Commons AttributionNonCommercial 4.0 International License (https://creativecommons.org/licenses/by-nc/4.0/), which permits unrestricted use, distribution, and reproduction in any medium for non-commercial purposes, provided the original author and source are credited, a link to the CC License is provided, and changes - if any - are indicated. 
Bats (forming the second largest order of mammals: Chiroptera) are well known for their widespread occurrence, migratory habit, frequently high population densities (especially during hibernation and nursing) and association with urban habitats (Dietz et al., 2009). In this way they are regarded as epidemiologically important, i.e. incriminated as reservoirs and carriers of viruses, bacteria and parasites of medical and veterinary significance (Klimpel and Mehlhorn, 2014). Accordingly, during the past years studies on bats and the pathogens they may harbour attracted great attention in the scientific world.

Babesia species (Apicomplexa: Piroplasmida) are tick-borne, intraerythrocytic protozoan parasites, with more than 100 species described from birds and mammals (Hunfeld et al., 2008). In this genus only B. vesperuginis is known to infect bats. Babesia vesperuginis elicits pathophysiological changes in bats, justifying its pathogenic nature (Gardner and Molyneux, 1987). The likely vector of $B$. vesperuginis is the soft tick Argas vespertilionis (Gardner and Molyneux, 1987).

Recently, the phylogenetic position of $B$. vesperuginis has been discussed, based on molecular analyses of its various isolates by two methods, i.e. PCR amplification and sequencing part of a nuclear (18S rRNA) gene (Hornok et al., 2016) and a mitochondrial marker [cytochrome $c$ oxidase subunit 1 (coxl)] (Corduneanu et al., 2017). Both studies indicated that phylogenetically $B$. vesperuginis aligns outside theileriae, but (as reflected by its name) within the clade of Babesia spp., as a sister group to other members of this genus. However, while results of these studies implied that $B$. vesperuginis is an important and unique species in the phylogeny of Piroplasmida, members of other important piroplasm groups (Theileria equi, the genus Cytauxzoon, prototheilerids or Western Babesia group and B. microti group: Schreeg et al., 2016) were not included in the reported phylogenetic trees. Therefore, the aim of this study was to compare the above two genes of $B$. vesperuginis in a broader context, i.e. to phylogenetically analyse new cox 1 sequences of $B$. vesperuginis (amplified from the bat soft tick $A$. vespertilionis) in the context of all five piroplasm groups, and to perform these analyses with concatenated $18 \mathrm{~S}$ rRNA and coxl gene sequences.

\section{Materials and methods}

Four DNA samples were used, which were extracted from bat soft tick ( $A$. vespertilionis) larvae and were positive in the PCR, amplifying an approx. 500bp-long part of the 18S rRNA gene of Babesia/Theileria spp. as already reported (Hornok et al., 2017). Relevant soft tick larvae were collected from grey longeared bat (Plecotus austriacus) at the Dráva river, Szent Anna Lake, Hungary, on 20 July 2015. Ethical approval for bat capture was provided by the National Inspectorate for Environment, Nature and Water; the bat banding licence number is TMF-493/3/2005 (TG). 
The four DNA extracts were further tested with a conventional PCR that amplifies an approx. 950-bp fragment of the cytochrome $c$ oxidase subunit 1 (coxl) gene of Piroplasmida. This method was modified from Gou et al. (2012), with two sets of primers: Bab For1: (5'- ATW GGA TTY TAT ATG AGT AT3') and Bab_Rev1: (5'- ATA ATC WGG WAT YCT CCT TGG-3'), then Bab_For2: (5'-TCT CTW CAT GGW TTA ATT ATG ATA T-3') and Bab_Rev2: (5'- TAG CTC CAA TTG AHA RWA CAA AGT G-3'). The 25- $\mu$ l reaction mixture contained $2 \mu \mathrm{DNA}$ template, $12.5 \mu$ l Master Mix (PCRBIO Taq Mix Red), $1 \mu 1$ of both primers $(10 \mathrm{pmol} / \mu \mathrm{l})$ and $8.5 \mu \mathrm{l}$ water. The thermal profile included $1 \mathrm{~min}$ initial denaturation at $95^{\circ} \mathrm{C}$, followed by 35 cycles of denaturation at $95{ }^{\circ} \mathrm{C}$ for $15 \mathrm{~s}$, annealing at $45^{\circ} \mathrm{C}$ for $30 \mathrm{~s}$ and extension at $72{ }^{\circ} \mathrm{C}$ for $1 \mathrm{~min}$. The final extension was performed at $72^{\circ} \mathrm{C}$ for $10 \mathrm{~min}$. Purification and sequencing of the PCR products were done by Macrogen Europe (Amsterdam, The Netherlands). The obtained sequences were manually edited, then aligned and compared to GenBank sequences by BLASTN program (https:// blast.ncbi.nlm.nih.gov). The consensus sequence was submitted to GenBank (accession number: KY657243). BLASTN comparisons between B. vesperuginis and other piroplasms, as elaborated below, include species shown on the phylogenetic trees, except for T. bicornis (for which coxl sequence was not available, therefore it had to be omitted from the phylogenetic trees).

Phylogenetic analyses of twenty $18 \mathrm{~S}$ rRNA and coxl gene nucleotide sequences (including those of $B$. vesperuginis: $448 \mathrm{bp}$ and $900 \mathrm{bp}$ in length, respectively) were conducted in three steps. First, coxl sequences with nearly $100 \%$ coverage to that of $B$. vesperuginis were compared with the Maximum Likelihood method [Hasegawa-Kishino-Yano (HKY) model], with 1000 resamplings, by using MEGA version 6.0 (Fig. 1).

Next, for concatenated trees, the evolutionary history was inferred by using the Maximum Likelihood method based on the General Time Reversible model (Nei and Kumar, 2000). Initial trees for the heuristic search were obtained automatically by applying Neighbour-Join and BioNJ algorithms to a matrix of pairwise distances estimated using the Maximum Composite Likelihood (MCL) approach, and then selecting the topology with superior log likelihood value. The tree with the highest log likelihood (-13216.2045) was selected (Fig. 2). A discrete Gamma distribution was used to model evolutionary rate differences among sites $[5$ categories $(+\mathrm{G}$, parameter $=0.8921)]$. The rate variation model allowed for some sites to be evolutionarily invariable $[(+\mathrm{I}), 31.1701 \%$ sites $]$. There were a total of 1433 positions in the final dataset. Evolutionary analyses were conducted in MEGA7 (Kumar et al., 2016). 


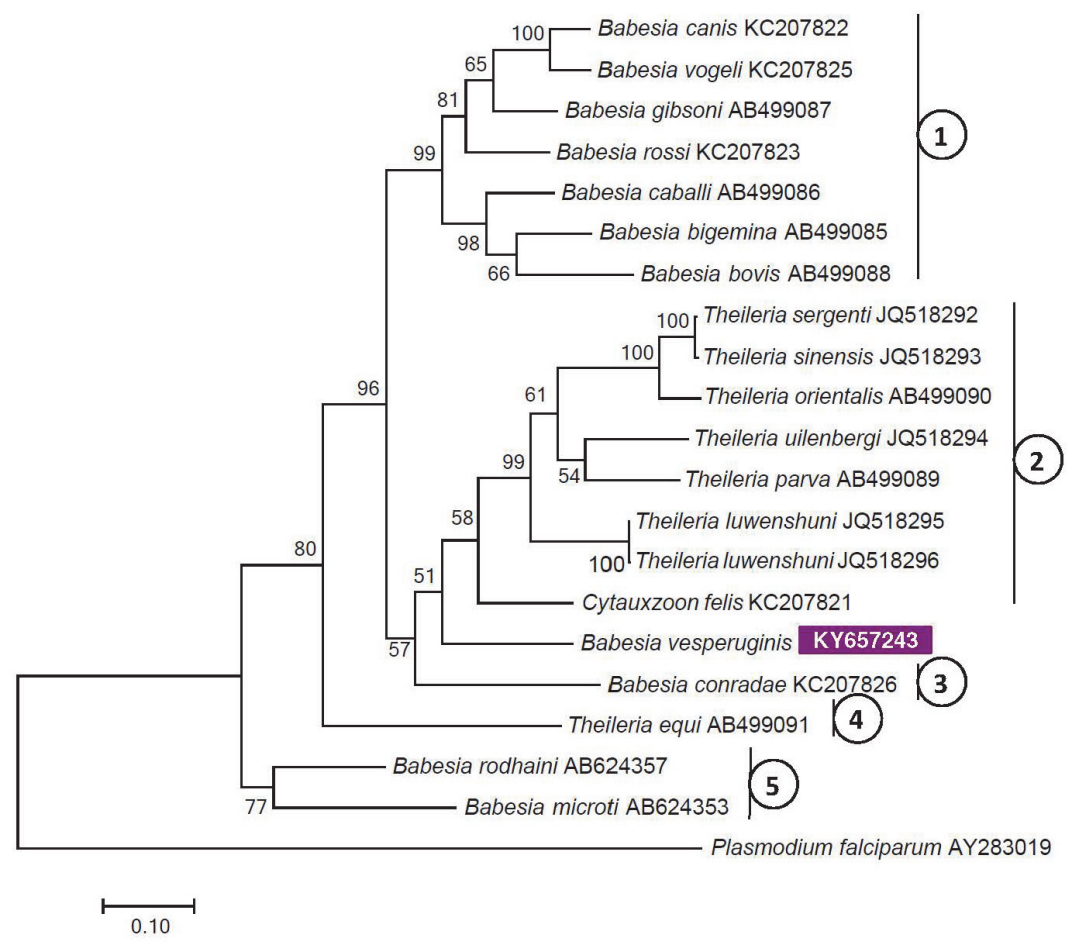

Fig. 1. Maximum likelihood tree of Piroplasmida based on coxl gene sequences, obtained with the Hasegawa-Kishino-Yano (HKY) model. Accession number of the Babesia vesperuginis sequence from this study is shown in inverse purple colour. The percentage of trees in which the associated taxa clustered together is shown next to the branches. Bootstrap values of $>50$ are shown. The tree is drawn to scale, with branch lengths measured in the number of substitutions per site. Numbers connected to vertical lines indicate category names (according to Schreeg et al., 2016) as follows: 1 - Babesia sensu stricto; 2 - Theileria (s.s.) and Cytauxzoon spp.; 3 - Western Babesia group; 4 - Theileria equi; 5 - Babesia microti group

An additional analysis was performed on the Phylogeny.fr platform (Dereeper et al., 2008; Dereeper et al., 2010) including the following steps. Sequences were aligned with MUSCLE (v3.8.31) (Edgar, 2004) configured for highest accuracy (MUSCLE with default settings). The phylogenetic tree (Fig. 3) was reconstructed using the Maximum Likelihood method implemented in the PhyML program (v3.1/3.0 aLRT) (Guindon and Gascuel, 2003; Anisimova and Gascuel, 2006). The HKY85 substitution model was selected assuming an estimated proportion of invariant sites (of 0.211 ) and 4 gamma-distributed rate categories to account for rate heterogeneity across sites. The gamma shape parameter was estimated directly from the data (gamma $=0.473)$. Reliability for internal branch was assessed using the aLRT test (SH-Like). Graphical representation and edition of the phylogenetic tree were performed with TreeDyn (v198.3) (Chevenet et al., 2006). 


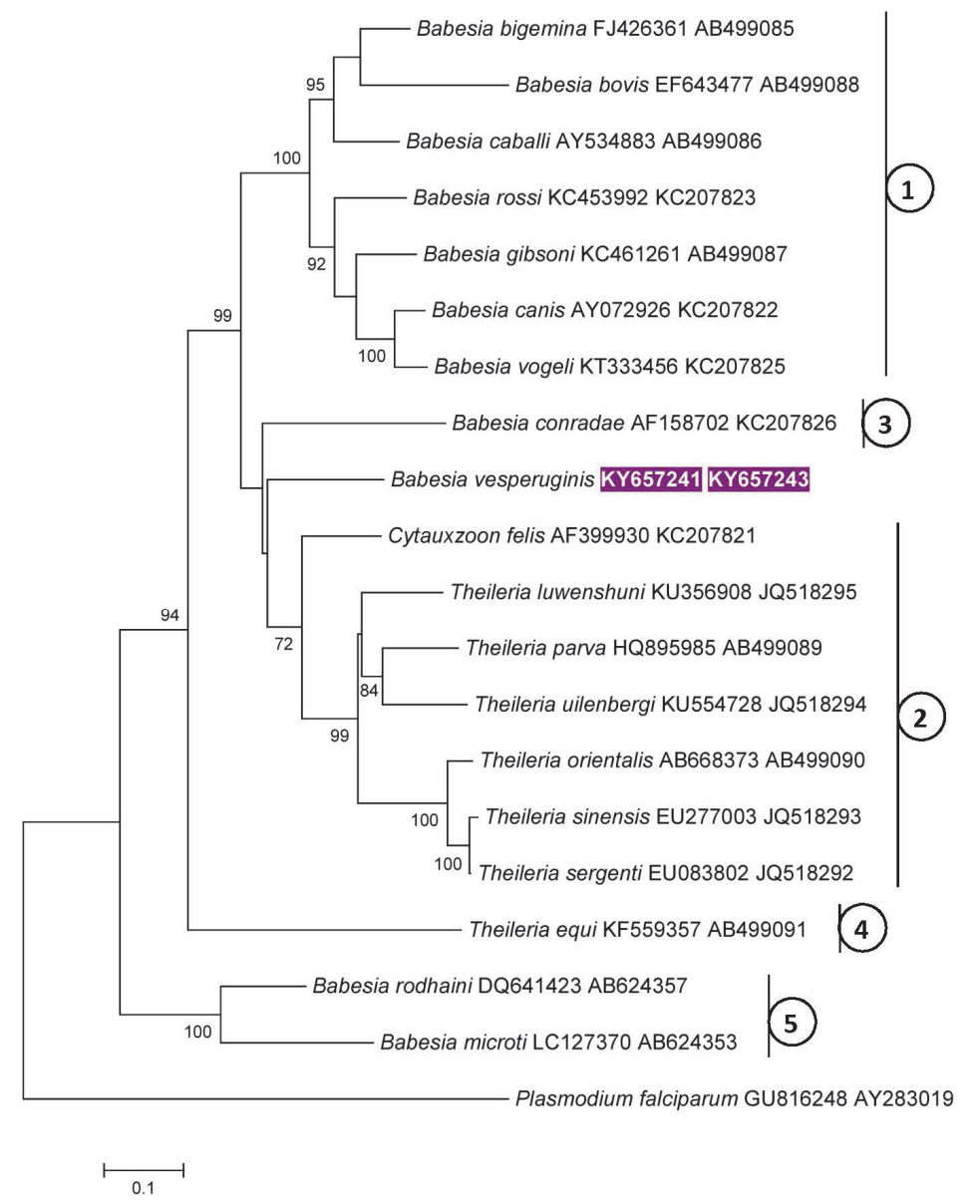

Fig. 2. Maximum likelihood tree of Piroplasmida based on concatenated cox 1 and 18S rRNA gene sequences (the GenBank accession numbers of which are shown in this order after the species name), obtained with the General Time Reversible (GTR) model. Accession number of Babesia vesperuginis sequence from this study is shown in inverse purple colour. The percentage of trees in which the associated taxa clustered together is shown next to the branches. Bootstrap values of $>70$ are shown. The tree is drawn to scale, with branch lengths measured in the number of substitutions per site. Numbers connected to vertical lines indicate category names (according to Schreeg et al., 2016) as follows: 1 - Babesia sensu stricto; 2 - Theileria (s.s.) and Cytauxzoon spp.; 3 - Western Babesia group; 4 - Theileria equi; 5 - Babesia microti group

\section{Results and discussion}

This is the first report on comprehensive phylogenetic analyses of $B$. vesperuginis, involving two genes. In addition, the cox 1 gene of $B$. vesperuginis was amplified for the first time from soft ticks, and also for the first time from a sam- 
ple collected in Hungary. All four samples yielded identical coxl sequences. This coxl sequence of $B$. vesperuginis (KY657243) had the highest similarity to that (KC207821) of Cytauxzoon felis (79.1\%, 709/896 bp), and less similarity to Babesia spp. [i.e. 74.9-77.6\% from B. bigemina (AB499085) to B. canis (KX712138), respectively] and Theileria spp. [i.e. 74.2-77.5\% from T. sinensis (JQ518293) to T. luwenshuni (JQ518295), respectively]. The number of gaps was up to 10 and 14 in comparison with Babesia and Theileria spp., but none in comparison with $C$. felis. The cox 1 sequences of $B$. vesperuginis and B. conradae (Western Babesia group) were only $73.2 \%$ (659/900 bp) similar, but in this alignment there were only two gaps.

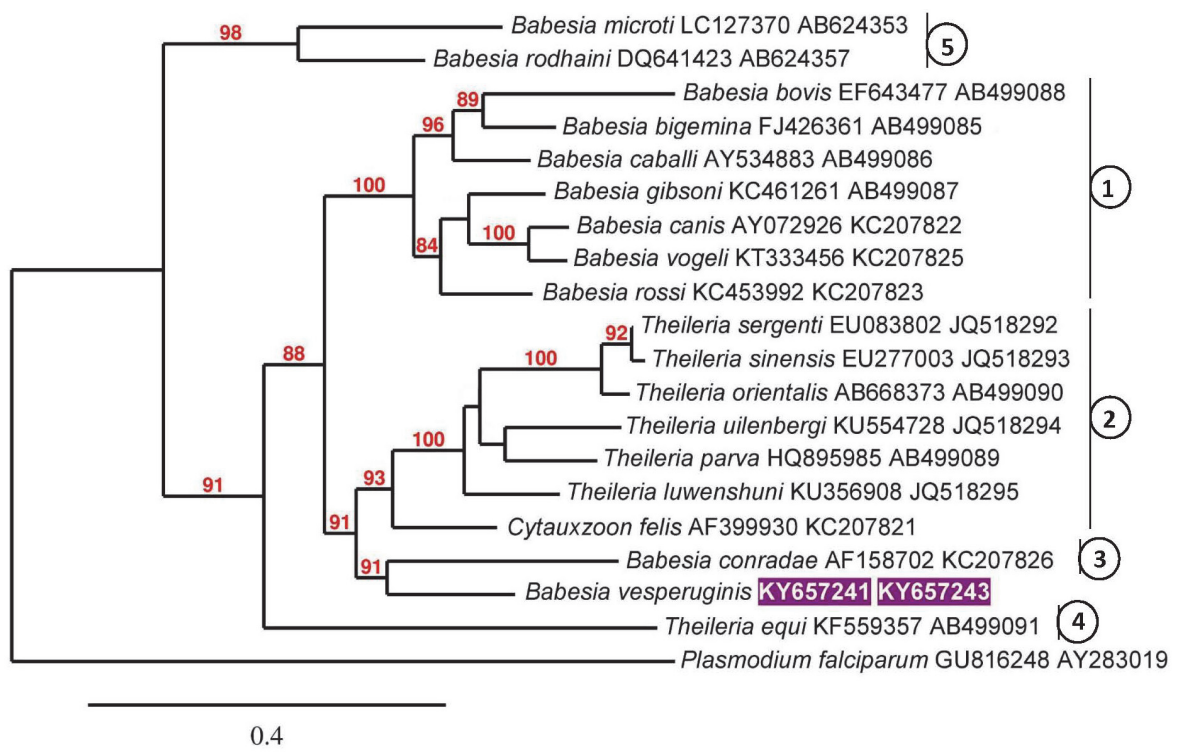

Fig. 3. Maximum likelihood tree of Piroplasmida based on concatenated coxl and 18S rRNA gene sequences (the GenBank accession numbers of which are shown in this order after the species name), obtained with the Hasegawa-Kishino-Yano (HKY) model. Accession number of Babesia vesperuginis sequence from this study is shown in inverse purple colour. The percentage of trees in which the associated taxa clustered together is shown next to the branches. Bootstrap values of $>70$ are shown. The tree is drawn to scale, with branch lengths measured in the number of substitutions per site. Numbers connected to vertical lines indicate category names (according to Schreeg et al., 2016) as follows: 1 - Babesia sensu stricto; 2 - Theileria (s.s.) and Cytauxzoon spp.; 3 - Western Babesia group; 4 - Theileria equi; 5 - Babesia microti group

This is in line with the highest similarity of $B$. vesperuginis $18 \mathrm{~S}$ rRNA sequence from $A$. vespertilionis (KY657241) with those of an 'outgroup Theileria', T. bicornis (AF499604: 422/455 bp $=92.7 \%$ identity); of the 'prototheilerid' B. conradae (AF158702: 416/450 bp =92.4\% identity); and of C. felis (AF399930: $409 / 450 \mathrm{bp}=90.9 \%$ identity) (sequences, category names are from: Criado- 
Fornelio et al., 2003; Lack et al., 2012; Hornok et al., 2017). On the other hand, the $B$. vesperuginis $18 \mathrm{~S}$ rRNA sequence had lower than $90 \%$ sequence identity with Babesia and Theileria spp. sensu stricto. In this decreasing order of sequence similarity, the number of gaps was relatively low in comparison with $T$. bicornis, B. conradae and C. felis (10, four and eight, respectively), whereas medium level (20-29) or high (28-58) in comparison with Theileria and Babesia spp., respectively.

The above sequence similarities and gap numbers were reflected by the topologies of phylogenetic trees. In particular, based on its coxl gene, $B$. vesperuginis clustered outside Babesia s.s. (with 96\% support) and close to C. felis and theileriae (Fig. 1). If $C$. felis was omitted from the analysis, $B$. vesperuginis remained a member of the phylogenetic group of Babesia s.s. (data not shown). This clearly argues against omitting $C$. felis (or other major piroplasmid categories) from phylogenetic analyses which serve to define the precise taxonomic status of $B$. vesperuginis.

Concerning phylogenetic analyses with concatenated coxl and 18S rRNA gene sequences performed here, both applied models achieved trees with similar overall topologies. In particular, $B$. vesperuginis clustered separately from Babesia spp., and this received a strong (Fig. 2: 99\%, Fig. 3: 88\%) support. In addition, $B$. vesperuginis clustered next to $B$. conradae and the phylogenetic group of Theileria spp. and C. felis (Fig. 2), or together with B. conradae in a sister group to the clade containing Theileriidae (Fig. 3).

Literature data on the developmental/morphological characteristics of relevant species support the above phylogenetic relationships of $B$. vesperuginis, i.e. its closer association with Theileria than with Babesia spp., and its closest association with $C$. felis and B. conradae. In particular, although the life cycle of $B$. vesperuginis is not completely known, it was reported to have quadrinucleated dividing forms ('tetrads') (Gardner and Molyneux, 1987). This is similar to what was reported in the case of $C$. felis (Glenn et al., 1982) and B. conradae (Kjemtrup et al., 2006). Unlike these, members of Babesia s.s. divide by binary fission in erythrocytes (Conrad et al., 2006).

The 18S rRNA gene is known to be a suitable marker for the estimation of phylogenetic relationships among Piroplasmida, and was therefore used in the great majority of studies to clarify the taxonomy of this group (Schreeg et al., 2016). However, using this gene alone resulted in some inconsistencies, and phylogenetic analyses do not agree on the taxonomic placement of certain Babesial Theileria spp. Consequently, adding to the $18 \mathrm{~S}$ rRNA gene, mitochondrial genome sequences [such as the cytochrome $c$ oxidase subunit I (coxl) gene] and their concatenation with the 18S rRNA sequences have proven to be useful for the elucidation of evolutionary relationships, delineating taxa and resolving Piroplasmida phylogeny (Gou et al., 2012; Schreeg et al., 2016). 
This is the first comprehensive analysis of this type, in which $B$. vesperuginis, a unique species was included. Taken together, phylogenetic trees in this study reflect the clustering pattern (separation of the same five piroplasmid groups) as recently reported in the case of phylogenetic trees based on coxl amino acid sequences or concatenated mitochondrial and 18S rRNA gene nucleotide sequences (Schreeg et al., 2016).

In conclusion, coxl sequence and phylogenetic analyses performed here clearly indicate that $B$. vesperuginis is not a member of the phylogenetic group Babesia s.s., but belongs to a separate clade, closely related to $C$. felis and $B$. conradae. Therefore, $B$. vesperuginis is a unique and taxonomically important species, which should be included in future studies serving to resolve the comprehensive phylogeny of Piroplasmida.

\section{Acknowledgements}

The study was organised in the framework of EurNegVec COST action TD1303. Financial support was provided by OTKA 115854 and the János Bolyai Research Scholarship of the Hungarian Academy of Sciences (to ADS). KB and MG is supported by the Lendület (Momentum) programme (LP2012-22) of the Hungarian Academy of Sciences. The publication of this research was supported by the 12190-4/2017/FEKUTSTRAT grant of the Hungarian Ministry of Human Resources.

\section{Competing interests}

The authors declare that they have no competing interests.

\section{References}

Anisimova, M. and Gascuel, O. (2006): Approximate likelihood ratio test for branches: A fast, accurate and powerful alternative. Syst. Biol. 55, 539-552.

Chevenet, F., Brun, C., Banuls, A. L., Jacq, B. and Chisten, R. (2006): TreeDyn: towards dynamic graphics and annotations for analyses of trees. BMC Bioinformatics 7, 439.

Conrad, P. A., Kjemtrup, A. M., Carreno, R. A., Thomford, J., Wainwright, K., Eberhard, M., Quick, R., Telford, S. R. 3rd and Herwaldt, B. L. (2006): Description of Babesia duncani n.sp. (Apicomplexa: Babesiidae) from humans and its differentiation from other piroplasms. Int. J. Parasitol. 36, 779-789.

Corduneanu, A., Hrazdilová, K., Sándor, A. D., Matei, I. A., Ionică, A. M., Barti, L., Ciocănău, M. A., Măntoiu, D. S., Coroiu., I., Hornok, S., Fuehrer, H. P., Leitner, N., Bagó, Z., Stefke, K., Modrý, D. and Mihalca, A. D. (2017): Babesia vesperuginis, a neglected piroplasmid: new host and geographical records, and phylogenetic relations. Parasit. Vectors 10, 598.

Criado-Fornelio, A., Martinez-Marcos, A., Buling-Sarana, A. and Barba-Carretero, J. C. (2003): Molecular studies on Babesia, Theileria and Hepatozoon in southern Europe. Part II. Phylogenetic analysis and evolutionary history. Vet. Parasitol. 114, 173-194. 
Dereeper, A., Audic, S., Claverie, J. M. and Blanc, G. (2010): BLAST-EXPLORER helps you building datasets for phylogenetic analysis. BMC Evol. Biol. 10, 8 .

Dereeper, A., Guignon, V., Blanc, G., Audic, S., Buffet, S., Chevenet, F., Dufayard, J. F., Guindon, S., Lefort, V., Lescot, M., Claverie, J. M. and Gascuel, O. (2008): Phylogeny.fr: robust phylogenetic analysis for the non-specialist. Nucleic Acids Res. 36, W465-9.

Dietz, C., von Helversen, O. and Nill, D. (2009): Bats of Britain, Europe and northwest Africa. A. C. Black Publishers Ltd., London. 406 pp.

Edgar, R. C. (2004): MUSCLE: multiple sequence alignment with high accuracy and high throughput. Nucleic Acids Res. 32, 1792-1797.

Gardner, R. A. and Molyneux, D. H. (1987): Babesia vesperuginis: natural and experimental infections in British bats Microchiroptera. Parasitol. 95, 461-469.

Glenn, B. L., Rolley, R. E. and Kocan, A. A. (1982): Cytauxzoon-like piroplasms in erythrocytes of wild-trapped bobcats in Oklahoma. J. Am. Vet. Med. Assoc. 181, 1251-1253.

Gou, H., Guan, G., Liu, A., Ma, M., Xu, Z., Liu, Z., Ren, Q., Li, Y., Yang, J., Chen, Z., Yin, H. and Luo, J. (2012): A DNA barcode for Piroplasmea. Acta Tropica 124, 92-97.

Guindon, S. and Gascuel, O. (2003): A simple, fast, and accurate algorithm to estimate large phylogenies by maximum likelihood. Syst. Biol. 52, 696-704.

Hornok, S., Szőke, K., Görföl, T., Földvári, G., Tu, V. T., Takács, N., Kontschán, J., Sándor, A. D., Estók, P., Epis, S., Boldogh, S., Kováts, D. and Wang, Y. (2017): Molecular investigations of the bat tick Argas vespertilionis (Ixodida: Argasidae) and Babesia vesperuginis (Apicomplexa: Piroplasmida) reflect 'bat connection' between Central Europe and Central Asia. Exp. Appl. Acarol. 72, 69-77.

Hornok, S., Szőke, K., Kováts, D., Estók, P., Görföl, T., Takács, N., Kontschán, J., Földvári, G., Barti, L., Corduneanu, A. and Sándor, A. D. (2016): DNA of piroplasms of ruminants and dogs in ixodid bat ticks. PLoS One 11, e0167735.

Hunfeld, K. P., Hildebrandt, A. and Gray, J. S. (2008): Babesiosis: Recent insights into an ancient disease. Int. J. Parasitol. 38, 1219-1237.

Kjemtrup, A. M., Wainwright, K., Miller, M., Penzhorn, B. L. and Carreno, R. A. (2006): Babesia conradae sp. nov., a small canine Babesia identified in California. Vet. Parasitol. 138, $103-111$.

Klimpel, S. and Mehlhorn, H. (2014): Bats (Chiroptera) as vectors of diseases and parasites: facts and myths. First edition. Springer, Berlin, Heidelberg. 187 pp.

Kumar, S., Stecher, G. and Tamura, K. (2016): MEGA7: Molecular Evolutionary Genetics Analysis Version 7.0 for Bigger Datasets. Mol. Biol. Evol. 33, 1870-1874.

Lack, J. B., Reichard, M. V. and Van Den Bussche, R. A. (2012): Phylogeny and evolution of the Piroplasmida as inferred from 18S rRNA sequences. Int. J. Parasitol. 42, 353-363.

Nei, M. and Kumar, S. (eds) (2000): Molecular Evolution and Phylogenetics. Oxford University Press, New York. 333 pp.

Schreeg, M. E., Marr, H. S., Tarigo, J. L., Cohn, L. A., Bird, D. M., Scholl, E. H., Levy, M. G., Wiegmann, B. M. and Birkenheuer, A. J. (2016): Mitochondrial genome sequences and structures aid in the resolution of Piroplasmida phylogeny. PLoS One 11, e0165702. 